\title{
O estresse como possível fator desencadeante de surtos de Esclerose Múltipla de acordo com 48 pacientes
}

\section{Stress as a possible triggering factor for relapses in Multiple Sclerosis according to 48 patients}

\author{
Ana Cláudia Pimenta Barbosa ${ }^{1}$, Luciane Oliveira Amaral', Verônica dos Santos Coelho ${ }^{1}$, \\ Yára Dadalti Fragoso².
}

\section{RESUMO}

Vários relatos da literatura sugerem que situações estressantes poderiam estar relacionadas a surtos de esclerose múltipla (EM). Os autores tiveram a impressão que muitos dos pacientes com EM relacionavam seus surtos a situações de estresse. Para avaliação desta impressão, entrevistamos individual e retrospectivamente um grupo de pacientes portadores de EM do tipo remitente-receorrente (EMRR). Os pacientes podiam fornecer livremente as informações, sem serem induzidos. Este grupo de 48 pacientes portadores de EMRR se constituía de 37 mulheres e 11 homens, com idades variando entre 14 e 64 anos. O diagnóstico definitivo de EMRR havia sido feito entre um e 24 anos (média $=7$ anos). Durante este período, o número de surtos variou entre dois e 12 (média $=4$ ). Estresse foi mencionado como principal causa de exacerbações por 27 pacientes, e como causa exclusiva por 18 pacientes. Infecções virais foram mencionadas como causa de surto por sete pacientes, calor excessivo por dois, enxaqueca particularmente forte por dois, gravidez por uma, depressão por uma e poluição ambiental por um paciente. A identificação de fatores desencadeantes não se relacionou à duração da doença, número de surtos, nível educacional, sexo ou idade dos pacientes. $56 \%$ de nossos pacientes espontaneamente mencionaram que situações de estresse eram o principal fator desencadeante de surtos de EM. Estudos prospectivos desta natureza, preferencialmente envolvendo diversos centros de atendimento de EM, necessitam ser realizados com um grande número de pacientes.

Unitermos: Esclerose múltipla, Estresse, Infecção.

\section{SUMMARY}

Various reports in the literature suggest that stressful life events may be related to relapses of Multiple Sclerosis (MS). We gained the impression that many of our patients related their bouts of MS to particularly stressful life situations. We have therefore performed a retrospective evaluation by means of individual interviews with patients suffering from relapsing-remitting MS (RRMS). The patients were free to volunteer

Trabalho realizado:CEREM Litoral Paulista \& Departamento de Neurologia, Faculdade de Medicina UNIMES, Santos, SP, Brasil. 
information about triggering factors, without being induced. This group of 48 patients suffering from RRMS consisted of 37 women and 11 men, aged 14 to 64 years. The definite diagnosis of RRMS had first been made one to 24 years ago (average $=7$ years). Since then, the number of bouts had varied from two to 12 (average $=4$ ). Stress was mentioned as the main cause of exacerbations by 27 patients, being the only cause for 18 patients. Viral infections were mentioned as possible cause by seven patients, hot weather by two, severe migraine by two, pregnancy by one, depression by one and environmental pollution by one. The identification of triggering factors did not relate to duration of disease, number of bouts, educational level, gender or age. $56 \%$ of our RRMS patients spontaneously mentioned stressful life events as important triggering factors for bouts of MS. Prospective studies of this nature, preferably involving several MS centers, should be carried out with a much larger number of patients.

Keywords: Multiple sclerosis, Stress, Infection.

\section{INTRODUÇÃO}

Esclerose Múltipla (EM) é uma doença autoimune, desmielinizante do sistema nervoso central. Em seu início, é comum o aparecimento de surtos isolados de desmielinização, que se manifestam por sinais e sintomas relacionados às vias afetadas, seguidos de recuperação funcional total ou parcial.

Frente a um novo surto de exacerbação da doença, muitos pacientes tentam relacioná-los a situações que os precederam, criando assim uma relação causa-efeito. Charcot já relatava, há mais de um século, que situações pessoais de súbita tristeza e de mudanças sociais poderiam ser desencadeantes de surtos ${ }^{1}$. É comum observarmos na prática clínica diária que os pacientes portadores de EM relatam situações de estresse como desencadeantes de piora clínica. No entanto, é necessário considerar que, apesar de uma possível relação entre o sistema imunológico e o estresse ${ }^{2,3}$, não é fácil classificar o tipo ou o efeito do estresse sobre um determinado indivíduo. Alguns estudos mais antigos não mostraram relação entre o estresse e os surtos de EM ${ }^{4,5}$. Estudos mais atuais, relativamente bem conduzidos, mostraram uma possível relação entre os fatos ${ }^{6-12}$. É interessante inclusive notar que um estudo mostrou que o estresse excessivo e contínuo de uma situação de guerra, não mostrou relação com exacerbação de surtos ${ }^{13}$. Embora este último estudo seja de duração relativamente curta, os achados foram interessantes, uma vez que parecia haver mesmo uma relação inversa entre o estresse intenso e crônico com a presença de novos surtos da doença. A possibilidade da liberação de cortisol endógeno mediante tais situações foi aventada como fator protetor de surtos durante o estresse ${ }^{14}$. No entanto, outro estudo recente mostrou uma nítida relação entre o estresse da perda de um filho menores de 18 anos e surtos de $\mathrm{EM}^{15}$. Continua portanto aberta à questão da relação entre estresse e surtos de EM.

Avaliamos um grupo de pacientes brasileiros com a finalidade de observar uma possível relação entre fatores desencadeantes e surtos de EM em nossa população.

\section{MÉTODOS}

Pacientes cadastrados no Centro de Referência para Diagnóstico e Tratamento de Esclerose Múltipla do Litoral Paulista (Universidade Metropolitana de Santos e DIR XIX da Secretaria do Estado da Saúde de SP) foram individualmente entrevistados como parte da anamnese. Todos os pacientes entrevistados apresentavam a forma remitente-recorrente de EM (EMRR). Os pacientes podiam fornecer livremente as informações, sem serem induzidos. A entrevista não oferecia alternativas ou opções, sendo os dados obtidos durante a história clínica. Este grupo consistia de 48 pacientes portadores de EMRR, sendo 37 mulheres e 11 homens, com idades variando entre 14 e 64 anos (média $=42$ anos). O tempo de educação formal destes pacientes era, em média, de 12 anos (variação $=3$ a 16 anos). O diagnóstico definitivo de EMRR havia sido feito entre um e 24 
anos (média $=7$ anos). Durante este período, o número de surtos variou entre dois e 12 (média = 4). A cada paciente foi perguntado se os surtos foram precedidos por algum nítido fator que poderia ser considerado como causal, com intervalo máximo de um mês entre tal acontecimento e as manifestações clínicas.

\section{RESULTADOS}

Vinte e sete pacientes (56\%) mencionaram que, quando algum fator podia ser identificado como possível desencadeante de surto, este fator parecia ser o estresse. Dezoito pacientes referiam que seus surtos haviam sido desencadeados por situações de estresse moderado a intenso, como perda do próprio emprego ou do emprego do cônjuge, dívidas acumuladas, divórcio, gravidez de filha menor, uso de drogas por filhos adolescentes, e doença grave, cirurgia ou morte de familiares ou amigos próximos. Estes 18 pacientes não se lembravam de outros fatores que pudessem estar relacionados a surtos da doença. Outros nove pacientes relatavam que além do estresse, outros fatores pareciam ter sido desencadeantes de seus surtos, tais como infecções virais, gravidez, e fortes crises de migrânea que duraram vários dias.

Oito pacientes referiam que seus surtos tinham se relacionado exclusivamente a infecções virais (cinco pacientes), calor excessivo (dois pacientes), poluentes ambientais (um paciente) e longo período de depressão (uma paciente).

Treze pacientes não relacionavam seus surtos a qualquer fator desencadeante.

A identificação de possíveis fatores desencadeantes de surtos não se relacionou a idade, sexo, nível cultural do paciente, tempo de doença ou número de surtos.

\section{DISCUSSÃO}

Estudos da relação entre estresse e surtos de EM podem, no máximo, apresentar evidência classe II. A falta de um modelo biológico de EM pura em situação de estresse, e a ausência da definição clara de "estesse" nas publicações sobre o tema, apresentam-se como falhas metodo- lógicas que dificultam a análise dos achados ${ }^{16-19}$. Além disso, o viés criado por entrevistas retrospectivas diminui a credibilidade dos achados de uma possível relação entre os fatos.

O presente trabalho apresenta as mesmas dificuldades metodológicas e se propõe apenas a discutir a impressão de um grupo de pacientes brasileiros. Apesar de todas as críticas aos métodos utilizados por trabalhos nesta linha, não se pode descartar uma observação clínica comum a diversos investigadores. Em estudos publicados por diversos autores e revisados por Goodin ${ }^{16}$, $56,8 \%$ a $79 \%$ dos pacientes portadores de EM relacionavam estresse aos surtos. A meta-análise de 20 trabalhos no tema, publicada em 2004 por Mohr ${ }^{17}$, também sugere associação, embora de fraca evidência, entre os eventos estressantes e os surtos de EM. No presente estudo, observamos que $56 \%$ de nossos pacientes espontaneamente relacionavam estresse a surtos de EM, dado semelhante aos dos autores estrangeiros. A exemplo dos casos relatados por outros investigadores, nossos pacientes também relacionaram outros fatores aos surtos, porém nenhum deles foi mencionado tão freqüentemente como o estresse.

A proposta de avaliação de mudanças de imagem na ressonância magnética (RNM) dos pacientes portadores de EM em situações de estresse foi aventada por Mohr em $2000^{20}$. Nos casos estudados, foram observadas novas lesões captantes de gadolíneo à RNM até 8 semanas após situações estressantes, mas não houve relação com surtos da doença. Infelizmente, tal método de investigação é excessivamente caro e não poderia ser executado de forma prática em nossa rotina de atendimento.

Os autores acreditam que pacientes brasileiros portadores de EM deveriam ser estudados prospectivamente em diversos centros universitários de diagnóstico e tratamento de EM. Um modelo adequado de avaliação seria aquele utilizado por Buljevac et al em $2003{ }^{10}$, avaliando porém uma população muito maior de pacientes, evitando assim parte das dificuldades naquele estudo, causadas pelo alto índice de pacientes perdidos ao seguimento. Apesar das críticas ao trabalho de Buljevac e colaboradores ${ }^{18,19,21}$, o modelo de uma avaliação prospectiva cuidadosa 
é o único que permite concluir o que Charcot já mencionava há mais de 100 anos $^{1}$ e que nos parece ser a opinião de diversos profissionais que trabalham com EM. Uma avaliação clínica desta natureza pode ser feita de forma prospectiva, incluindo vários centros universitários, e seguindo um grande número de pacientes por um longo período.

\section{REFERÊNCIAS BIBLIOGRÁFICAS}

1- Charcot JM. Lectures on diseases of the nervous system. London: New Sydenham Society; 1877.

2- Van Nort JM. Multiple sclerosis : an altered immune response or an altered stress response. J Mol Med 1996; 74: 285-296.

3- Fassbender K, Schmidt R, Mößner R, et al. Mood disorders and dysfunction of hypothalamic-pituitary-adrenal axis in multiple sclerosis: association with cerebral inflammation. Arch Neurol 1998; 55: 66-72.

4-Pratt RTC. An investigation of the psychiatric aspects of disseminated sclerosis. J Neurol Neurosurg Psychiatry 1951; 14: 326-336.

5-Antonovsky A, Leibowitz U, Medalie JM, et al. Reappraisal of possible etiologic factors in multiple sclerosis. Am J Public Health 1968; 58: 836-848.

6- Warren S, Greenhill S, Warren KG. Emotional stress and the development of multiple sclerosis : case-control evidence of a relationship. J Chronic Dis 1982; 35: 821-831.

7- Franklin GM, Nelson LM, Heaton RK, et al. Stress and its relationship to acute exacerbations in multiple sclerosis. J Neurol Rehab 1988; 2: 7-11.

8- Grant I, Brown GW, Harris T, et al. Severely threatening events and marked life difficulties preceding onset or exacerbation of multiple sclerosis. J Neurol Neurosurg Psychiatry 1989; 52: 8-13

9- Warren W, Warren KG, Cockerill R. Emotional stress and coping in multiple sclerosis (MS) exacerbations. J Psychosomat Res 1991; 35: 37-47.

10- Buljevac D, Hop WC, Reedeker W, Janssens AC, van der Meche FG, van Doorn PA, et al. Self reported stressful life events and exacerbations in multiple sclerosis: prospective study. BMJ 2003; 327: 646.

11- Ackerman KD, Stover A, Heyman R, Anderson BP, Houck PR, Frank E, et al. Relationship between cardiovascular reactivity, stressful life events, and multiple sclerosis disease activity. Brain Behavior Immun 2003; 17: 141-151.
12- Schwartz CE, Foley FW, Rao SM, Bernardin LJ, Lee H, Genderson MW. Stress and course of disease in multiple sclerosis. Behav Med 1999; 25: 110-116

13- Nisipeanu P, Korczyn AD. Psychological stress as a risk factor for exacerbation in multiple sclerosis. Neurology 1993; 43: 1311-1312.

14- Munck A, Naray-Fejes-Toth A. Glucocorticoids and stress: permissive and suppressive actions. Ann N Y Acad Sci 1994; 746: 115-133.

15- Li J, Johansen C, Brønnum-Hansen H, Stenager E, Koch-Henriksen N, Olsen J. The risk of multiple sclerosis in bereaved parents. Neurology 2004; 62: 726-729.

16- Goodin DS, Ebers GC, Johnson KP, Rodriguez M, Sibley WA, Wolinsky JS. The relationship of MS to physical trauma and psychological stress. Neurology 1999; 52: 1737-1745.

17- Mohr DC, Hart SL, Julian L, Cox D, Pelletier D. Association between stressful life events and exacerbation in multiple sclerosis: a meta-analysis BMJ 2004; 328: 731

18- Galea I, Gidron Y, Newman TA. Stressful life events increase exacerbations in multiple sclerosis: far from proven. BMJ 2003; http://bmj.com/cgi/ eletters/327/7416/646\#37564

19- Galea I, Newman TA, Gidron Y. Stress and exacerbations in multiple sclerosis: Whether stress triggers relapses remains a conundrum. BMJ 2004; 328: 287.

20- Mohr DC, Goodkin DE, Bacchetti P, Boudewyn AC, Huang L, Marrietta P, Cheuk W, Dee B. Psychological stress and the subsequent appearance of new brain MRI lesions in MS. Neurology 2000; 55: 55-61.

21-Barnes D. Inconsequential research. BMJ 2003; http://bmj.com/cgi/eletters/327/7416/646 \#36922 